\title{
Acquisition of Nicotine Self-Administration in Adolescent Rats Given Prolonged Access to the Drug
}

\author{
Hao Chen', Shannon G Matta' and Burt M Sharp*,' \\ 'Department of Pharmacology, University of Tennessee Health Science Center, Memphis, TN, USA
}

As most human tobacco use begins during adolescence and ongoing development of the adolescent central nervous system could affect acquisition of nicotine self-administration (SA), our established rat SA procedure was modified to study adolescent acquisition of SA with prolonged access to nicotine $(23 \mathrm{~h} /$ day). Postnatal age $43-45$ female Lewis rats, without prior shaping, conditioning, or food deprivation, were housed in operant chambers equipped with two levers; pressing the active lever triggered an i.v. injection of nicotine. By the IOth day of SA, rats receiving $7.5,15,30$, or $60 \mu \mathrm{g} / \mathrm{kg} /$ injection nicotine (free base) obtained $23 \pm 16,50 \pm 8$, $65 \pm 8$, or $48 \pm 5$ injections (mean $\pm \mathrm{SE}$ ), respectively. In the $30 \mu \mathrm{g} / \mathrm{kg} /$ injection group, active: inactive ratio was greater than 2 after SA day 4; $92 \%$ of injections occurred during the 12-h active (dark) phase of the light cycle. Main effects (analysis of variance) were shown for day and lever (ie active vs inactive) $(p<0.00 \mathrm{I})$. Adolescent males showed similar dose-dependent nicotine SA. With the increasing workload imposed by raising the fixed ratio (FR), less nicotine was self-administered at FR 5 and 7 compared to FR I and 3. In comparison to adult females selfadministering $30 \mu \mathrm{g} / \mathrm{kg} /$ injection of nicotine at FR I, adolescents acquired nicotine SA at an accelerated rate $(p<0.05)$ and received a greater number of injections $(p<0.05)$ by day 10 . In conclusion, when given prolonged access to the drug, both female and male adolescent Lewis rats rapidly acquire nicotine SA within the dosage range and FR constraints previously observed in adult Lewis rats. However, adolescent females acquired the behavior more rapidly and attained higher levels of stable nicotine SA than adults.

Neuropsychopharmacology (2007) 32, 700-709. doi:I0.1038/sj.npp. I 30 I I35; published online I4 June 2006

Keywords: nicotine; self-administration; adolescence; gender; development; operant

\section{INTRODUCTION}

Adolescence is a phase of brain growth and development in which individuals seek new experiences and higher levels of rewarding stimulation by engaging in risk-taking and novelty-seeking behaviors. Although these behaviors can have certain adaptive benefits in facilitating independence, they can also predispose adolescents to initiate drug abuse (Spear, 2000; Kelley et al, 2004). In fact, the great majority of tobacco use begins during adolescence; approximately $80 \%$ of adult tobacco users in USA reported that they first used tobacco before age 18, and $60 \%$ used before age 14 (Glynn et al, 1993; Eissenberg and Balster, 2000). In 2002, 15.2\% of youth (age 12-17 years) reported use of tobacco products; more than $30 \%$ of these individuals reported smoking cigarettes daily (SAMHSA, 2002). Initiation of smoking during adolescence is associated with higher daily consumption of cigarettes (Chen and Millar, 1998; Everett et al,

* Correspondence: Dr BM Sharp, Department of Pharmacology, University of Tennessee Health Science Center, 874 Union Avenue, Room I15, Memphis, TN 38163, USA, Tel: + I 901448 6000, Fax: + I 901448 7206, E-mail: bsharp@utmem.edu

Received 17 January 2006; revised 30 March 2006; accepted 15 May 2006

Online publication: 23 May 2006 at http://www.acnp.org/citations/ Npp052306060023/default.pdf
1999; Fernandez et al, 1999) and lower probability of quitting in adulthood (Chen and Millar, 1998). Consequently, the earlier an individual starts, the more likely they will become a lifelong smoker (Rigotti, 1990).

Nicotine is the principal psychoactive agent in tobacco. It possesses reinforcing properties and is a powerful addictive agent (Stolerman and Jarvis, 1995). In animal studies, nicotine has been shown to induce locomotor activation and behavioral sensitization (Schoffelmeer et al, 2002), both of which model the neuroplasticity associated with psychostimulant drugs. More importantly, nicotine is self-administered by a variety of species, including mouse (Rasmussen and Swedberg, 1998), rat (Hanson et al, 1979; Cox et al, 1984; Corrigall and Coen, 1989; Donny et al, 1995; Valentine et al, 1997), dog (Risner and Goldberg, 1983), primate (Goldberg et al, 1981), and human (Jaffe and Kanzler, 1978; Rose et al, 2003), clearly demonstrating its reinforcing properties.

Despite strong epidemiological evidence for the onset of chronic cigarette smoking during adolescence, most animal studies of nicotine have focused on adults. As the final phase of neurodevelopment occurs during adolescence (Spear, 2000), nicotine may differentially affect the brains of adolescents compared to adults. It has been shown, for example, that adolescent brains are especially vulnerable to the neurotoxic effects of nicotine (Slotkin, 2002). Therefore, 
it has become increasingly important to develop models of adolescent nicotine self-administration (SA) in order to elucidate the unique effects of nicotine on neuronal function, neurochemistry, and behavior during the critical phase of adolescent brain development.

Many behavioral responses to nicotine differ between adolescent and adult rats. Adolescents are more sensitive to locomotor enhancement by acute (Schochet et al, 2004) and chronic nicotine (Faraday et al, 2001, 2003), and are less sensitive to locomotor suppression (Vastola et al, 2002). However, adolescents display less robust nicotine-induced locomotor sensitization (Schochet et al, 2004; Cruz et al, 2005) and cue conditioning (Schochet et al, 2004) compared to adult rats. Conflicting results have been reported for conditioned place preference. Depending on age (early vs late adolescence) (Belluzzi et al, 2004) and dose (Vastola et al, 2002; Belluzzi et al, 2004), adolescents show either enhanced or reduced conditioned place preference. In addition, sensitivity to the somatic aspects of nicotine withdrawal is decreased in adolescents (O'Dell et al, 2004) and persistent reductions in motor activity are still observed 2-6 weeks after withdrawal (Trauth et al, 2000a; Slawecki and Ehlers, 2002). These data, demonstrating the unique characteristics of the adolescent behavioral response to nicotine, underscore the importance of understanding nicotine SA during adolescence.

Recently, a limited access (1-3 h/day) model of nicotine $\mathrm{SA}$ in adolescent rats has been reported. Female rats that initiated nicotine SA during late adolescence (postnatal days (PN) 50-62) had higher levels of adult nicotine intake than cohorts starting to self-administer during adulthood (PN 80-85) (Levin et al, 2003). In addition, using a similar limited access model of nicotine SA, Belluzzi et al (2005) reported that early adolescent rats ( $P N$ 27) maintained nicotine SA only if acetaldehyde was included in the mixture, failing to do so when nicotine alone was provided.

Herein, we modified our established model (Valentine et al, 1997) of prolonged access $(23 \mathrm{~h} /$ day) to nicotine SA in adult rats in order to study the acquisition of nicotine SA during mid-adolescence, beginning at PN 40-42. Acquisition of nicotine SA was evaluated across a range of dosages in both genders of Lewis rats, which are genetically prone to self-administer nicotine and other drugs of abuse (Brower et al, 2002; Kosten and Ambrosio, 2002). We also determined the effects of increasing demand on adolescent female SA behavior, using an escalating fixed ratio (FR) schedule, and compared the acquisition of nicotine SA between female adolescent and adult rats. Using this model of prolonged access ( $23 \mathrm{~h} /$ day), these studies demonstrated that nicotine SA was reliably acquired by both female and male adolescents, within a dosage range similar to adults. Moreover, the adolescent FR response profile (FR 1-FR 7) was similar to that reported in adults (Brower et al, 2002). However, female adolescents acquired nicotine SA significantly more rapidly than adults and achieved higher levels of stable nicotine SA.

\section{MATERIALS AND METHODS}

Nicotine hydrogen tartrate was purchased from Sigma (St Louis, MO). Nicotine dosages ( $\mathrm{pH} \mathrm{7.2;} \mathrm{calculated} \mathrm{as} \mathrm{free}$ base), freshly prepared in heparinized saline for each cohort of animals, were calculated to deliver $7.5-60 \mu \mathrm{g} / \mathrm{kg}$ body weight (b.wt.) in a $50 \mu$ volume: these solutions were stored in foil-wrapped glass bottles at $4{ }^{\circ} \mathrm{C}$ for no longer than 10 days. Heparin was purchased from Henry Schein Inc. (Melville, NY) and Baytril from Bayer Corp. (Shawnee Mission, KS).

\section{Animals}

Adolescent Lewis rats (PN 33-35; Harlan, Indianapolis, IN) were given 7 days to recover from shipping and acclimation to a reversed 12:12-h light/dark cycle (lights off at 0930 hours). Standard rat chow and water were provided ad libitum throughout the experiments. All procedures were conducted in accordance with the NIH Guidelines Concerning the Care and Use of Laboratory Animals and were approved by the Animal Care and Use Committee of the University of Tennessee.

\section{Acquisition of Nicotine SA}

SA was performed according to our previously published protocol with some modifications (Valentine et al, 1997; Brower et al, 2002). Jugular catheters (PE 90 and silastic) were implanted on PN 40-42 and each rat was transferred to a home operant chamber (Coulbourn Instruments, Allentown, PA) inside a sound-attenuating environmental enclosure, where it resided for the duration of the experiment. Each operant chamber contained two horizontal levers positioned $4 \mathrm{~cm}$ above the floor. A green cue light located $1 \mathrm{~cm}$ above each lever was illuminated only when nicotine was available. Lever presses were recorded and syringe pumps were controlled by computers and interfaces located in an adjacent room, using L2T2 or Graphic State software (Coulbourn Instruments). Three days after recovery from jugular surgery, during which time rats received daily antibiotic injections (Baytril, $7.6 \mathrm{mg} / \mathrm{kg}$ b.wt. in $0.1 \mathrm{ml}$, i.v.) and hourly computer-driven aliquots $(50 \mu \mathrm{l})$ of heparin-containing heparinized saline $(200 \mathrm{IU} / \mathrm{ml})$, nicotine was first made available.

On the day that SA was initiated (SA day 1), one lever in the chamber was randomly designated as the active lever. Pressing the active lever elicited a computer-driven i.v. injection of nicotine delivered in $50 \mu$ l over $0.81 \mathrm{~s}$ through the jugular catheter. Pressing the alternate (inactive) lever had no programmed consequence. To avoid over-dosing, each injection was followed by a 7-s period during which the green cue light was extinguished and lever pressing was recorded, but nicotine was not injected.

The final hour of the lights-on cycle ( 0830 hours) was reserved for housekeeping tasks, such as replacing the nicotine with fresh solutions, performing animal husbandry, measuring b.wt., resetting the computer program, and downloading data. During this interval, the doors of the environmental enclosures were opened and green cue lights were turned off to signal to the rats that nicotine was unavailable. Levers were not retracted, and lever press activity was not recorded nor rewarded during this period. Patency of each jugular line was checked every 2-3 days. Rats with closed lines were excluded from data analysis. 


\section{Dose-Response Procedure}

Four groups of adolescent rats were used to study the acquisition of nicotine SA in females at dosages of 7.5 $(n=5), 15(n=6), 30(n=8)$, and $60(n=5) \mu \mathrm{g} / \mathrm{kg}$ b.wt. For comparison to the female data, three groups of male adolescent rats were used to evaluate potential genderdependent sensitivity to acquisition of nicotine SA at 7.5 $(n=5), 15(n=6)$, and $30(n=10) \mu \mathrm{g} / \mathrm{kg}$ b.wt. Animals were allowed to self-administer nicotine for 10 days.

\section{Escalating FR Schedule}

These experiments assessed the ability to maintain nicotine SA at escalating FR schedules, such that an increasing number of active lever presses was required to receive a single injection of nicotine. Female adolescent rats were allowed to initiate nicotine SA at an FR 1 schedule, in which each active lever press resulted in delivery of a single $50-\mu 1$ aliquot of $30 \mu \mathrm{g} / \mathrm{kg}$ b.wt. i.v. nicotine. This FR 1 schedule continued for 7 days and was then increased to FR 2 (3 days), FR 3 (5 days), FR 5 (5 days), and FR 7 (5 days).

\section{Nicotine SA in Adult Female Rats}

To compare nicotine SA in adolescent and adult rats, 10 adult female Lewis rats were allowed access to $30 \mu \mathrm{g} / \mathrm{kg} /$ injection of nicotine for 10 days. Our standard protocol (Valentine et al, 1997; Brower et al, 2002) for nicotine SA was used. Operant chambers were identical to those used for adolescent studies except that the height of the active lever was $4 \mathrm{~cm}$ for adolescents and $5 \mathrm{~cm}$ for adults.

\section{Statistics}

Data are presented as mean \pm standard error of mean (SEM). The difference in active and inactive lever presses was analyzed using repeated measures analysis of variance (ANOVA) for each nicotine dosage and gender, treating day and lever as within-subject variables. The effect of different nicotine dosages on the number of active lever presses was analyzed for each gender using repeated measures ANOVA, using day as a within-subjects variable and dosage as a between-subjects variable. Gender difference in the number of active lever presses during nicotine SA was analyzed using repeated measures ANOVA, treating day as a withinsubjects variable, whereas dosage and gender were between-subjects variables. The difference in the number of injections received during the last 2 days at each FR was analyzed by ANOVA. Comparison of the slopes of linear regressions (injection $\sim$ day) for adolescent $v s$ adult females was calculated according to Zar (1984). All other statistical analyses were performed using SPSS (SPSS Inc., Chicago, IL, USA). Statistical significance was assigned when $p<0.05$.

\section{RESULTS}

\section{Acquisition of Nicotine SA at Different Dosages in Female Adolescent Rats}

Beginning on PN 43-45, female adolescent rats were given access to i.v. nicotine for $23 \mathrm{~h}$ each day. At $7.5 \mu \mathrm{g} / \mathrm{kg}$ b.wt. nicotine (Figure 1a), only two rats showed an increasing number of daily active lever presses within 3-4 days of nicotine becoming available (dashed top line); inactive lever presses were unaffected. However, this active lever press behavior was not maintained; after reaching a peak on SA day 7 ( $149 \pm 25$ injections), it steadily declined to $48 \pm 38$ injections by SA day 10 . The other three rats in this group did not demonstrate any difference between active or inactive lever presses. Repeated measures ANOVA found no statistically significant difference between the levers $\left(\mathrm{F}_{1,8}=1.50, p>0.05\right)$ or the days $\left(\mathrm{F}_{9,40}=1.97, p>0.05\right)$ when all five animals were included in the analysis. Thus, only $40 \%$ of the rats in the $7.5 \mu \mathrm{g} / \mathrm{kg}$ dosage group showed some initial evidence of nicotine SA, which was not sustained (Figure 1a).

At nicotine dosages of 15,30 , and $60 \mu \mathrm{g} / \mathrm{kg}$, the number of active lever presses rapidly increased during the first few days that nicotine was available and stabilized, thereafter, particularly at 15 and $30 \mu \mathrm{g} / \mathrm{kg}$ (Figure $1 \mathrm{~b}-\mathrm{d}$ ). Throughout these experiments, inactive lever presses were unchanged. At all three dosages, the ratio of active to inactive presses was greater than 2 by SA day 4 and on all subsequent days. By SA day 10, active lever presses were $50 \pm 8,65 \pm 8$, and $48 \pm 5$ at these three dosages, respectively; inactive lever presses were $22 \pm 3,18 \pm 4$, and $11 \pm 4$, respectively. Repeated measures ANOVA showed main effects of lever $\left(\mathrm{F}_{1,10}=17.65, \quad p<0.01 ; \quad \mathrm{F}_{1,14}=37.35\right.$, $p<0.001 ; \mathrm{F}_{1,8}=7.98, p<0.05$ for the three nicotine dosages, respectively $)$ and day $\left(\mathrm{F}_{9,50}=11.53, \quad \mathrm{~F}_{9,70}=14.19\right.$, $\mathrm{F}_{9,40}=10.65$ for the three dosages, respectively; each at $p<0.001)$. In addition, there was an interaction between lever and day $\left(\mathrm{F}_{9,50}=4.82, \mathrm{~F}_{9,70}=12.43, \mathrm{~F}_{9,40}=7.64\right.$ for the three dosages, respectively; each at $p<0.001)$. These experiments demonstrate that adolescent female Lewis rats reliably self-administer nicotine at dosages between 15 and $60 \mu \mathrm{g} / \mathrm{kg}$. The characteristics of this behavior included the following: a progressive increase in active lever presses during the first few days of SA, stable active lever press levels of approximately 60-80/day after 6-7 days of access to nicotine SA, as well as a consistent and strong preference for the active lever throughout these experiments (Figure $1 \mathrm{~b}-\mathrm{d}$ ).

\section{Sensitivity to Nicotine during Acquisition of SA in Male Adolescent Rats: Comparison to Female Adolescents}

We evaluated whether the sensitivity of male adolescent rats to nicotine during acquisition of SA was different than females by testing three nicotine dosages $(7.5,15$, and $30 \mu \mathrm{g} /$ $\mathrm{kg}$ ). Similar to female adolescents, at $7.5 \mu \mathrm{g} / \mathrm{kg}$ nicotine, only two adolescent males showed a gradual increase in active lever presses that was maximal by SA day 7, but was not sustained thereafter (Figure 2a). However, the average level of active lever presses in these two male responders was considerably less than in the two female responders (Figure 1a). The other three male rats had similar active and inactive lever press activity throughout the 10 days of SA (Figure 2a). When all five animals were analyzed together, repeated measures ANOVA found no significant difference between the two levers $\left(\mathrm{F}_{1,8}=2.21, p>0.05\right)$ at nicotine $7.5 \mu \mathrm{g} / \mathrm{kg}$. However, there was an effect of day $\left(\mathrm{F}_{9,40}=3.36\right.$, $p<0.01)$. 


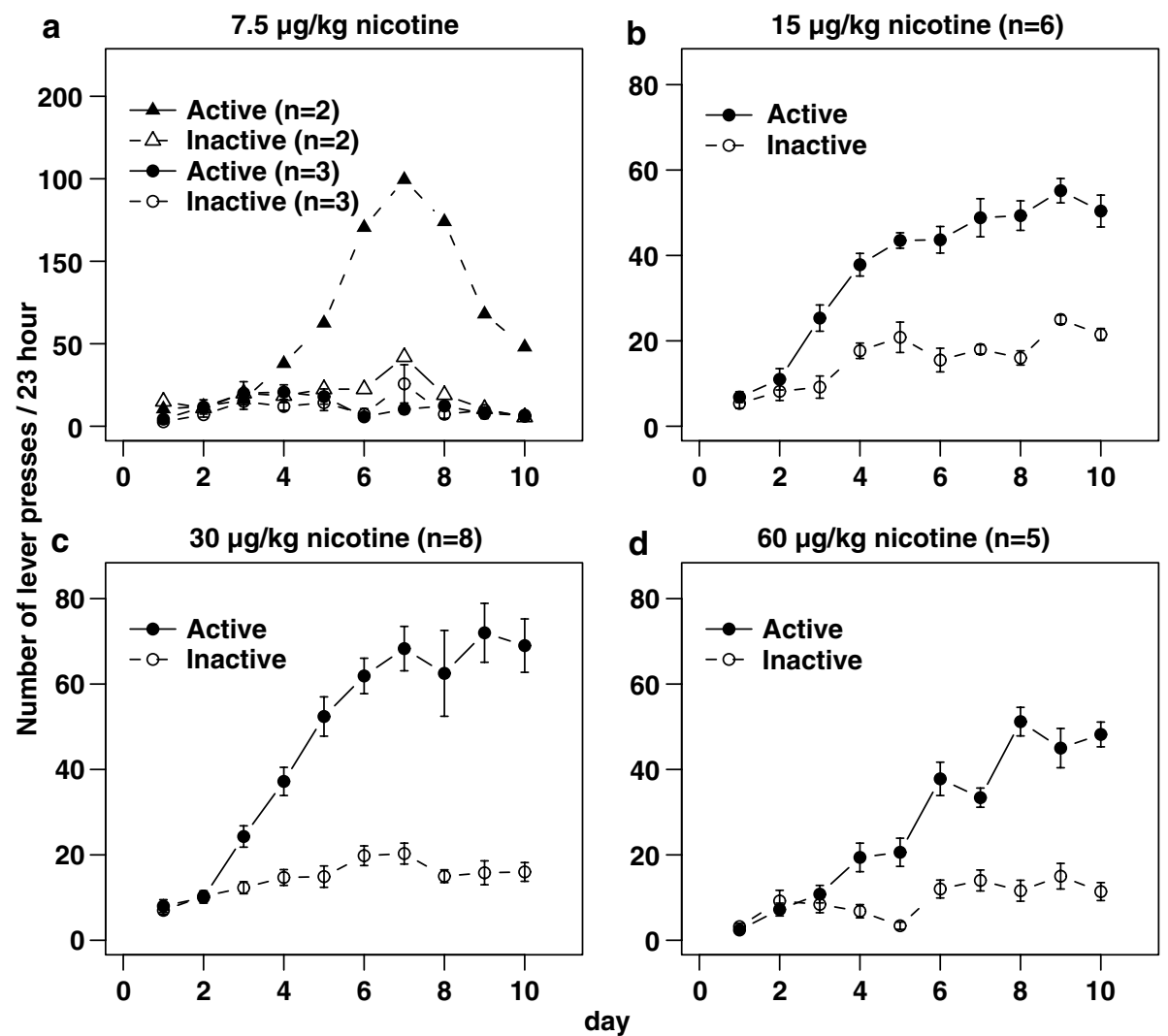

Figure I Dosage-dependent acquisition of nicotine SA in female adolescent Lewis rats. Female mid-adolescent rats (PN 43-45) were given access to selfadminister i.v. nicotine for $23 \mathrm{~h}$ each day. All data are presented as mean \pm standard error of mean (SEM). (a) At $7.5 \mu \mathrm{g} / \mathrm{kg}$ nicotine, only two of the five rats showed initial acquisition of nicotine SA, but it was not sustained. No statistically significant difference between the levers $(p>0.05)$ or the days $(p>0.05)$ was found when all five animals were included in repeated measure ANOVA. (b-d) At nicotine dosages of I5, 30, and 60 $\mu \mathrm{g} / \mathrm{kg}$, respectively, active lever presses rapidly increased during the first few days that nicotine was available, and stabilized thereafter. Inactive lever presses were unchanged throughout. At all three dosages, the ratio of active to inactive presses was greater than 2 after SA day 4 . Repeated measures ANOVA showed main effects of lever $(p<0.01,0.00 \mathrm{I}$, and 0.05 for the three dosages, respectively) and day ( $p<0.00 \mathrm{I}$ for all three dosages), and interaction between lever and day $(p<0.00 \mathrm{I}$ for all three dosages).

At 15 and $30 \mu \mathrm{g} / \mathrm{kg}$ nicotine, stable SA was achieved in male adolescents (Figure $2 \mathrm{~b}$ and $\mathrm{c}$ ). Active to inactive ratios were greater than 2 on SA day 2 and thereafter, although on SA day 7 the group receiving injections of $15 \mu \mathrm{g} / \mathrm{kg}$ had a ratio $=1.85$. By SA day 10 , active lever presses were $74 \pm 10$, and $56 \pm 6$ at the two dosages, respectively, and inactive lever presses were $24 \pm 3$ and $21 \pm 5$, respectively. Repeated measures ANOVA showed main effects of lever $\left(F_{1,10}=33.27\right.$ and $F_{1,18}=33.79$ for each dosage, respectively, both at $p<0.001)$ and day $\left(\mathrm{F}_{9,50}=8.51\right.$ and $\mathrm{F}_{9,90}=11.20$ for each dosage, respectively, both at $p<0.001)$. In addition, there was an interaction between lever and day $\left(\mathrm{F}_{9,50}=2.37, p<0.001\right.$ and $\mathrm{F}_{9,90}=8.06$, $p<0.05$ for each dosage, respectively). These data demonstrate that, similar to adolescent females, adolescent male Lewis rats reliably acquired nicotine SA within the dosage range of $15-30 \mu \mathrm{g} / \mathrm{kg}$. Together with the data showing that both adolescent males and females failed to acquire stable nicotine SA at the lowest dosage tested $(7.5 \mu \mathrm{g} / \mathrm{kg})$, these results indicate that the acquisition of nicotine $\mathrm{SA}$ in adolescent male and female Lewis rats shows no difference in sensitivity to nicotine dosage. In addition, comparison of the average number of active lever presses by male and female adolescents during the last 2 days at each dosage showed no effect of gender $\left(\mathrm{F}_{1,38}=0.46 ; p=0.50\right.$; repeated measures ANOVA).

\section{Effect of Nicotine Dosage on SA in Both Genders}

The effect of nicotine dosage on SA was evaluated by comparing the number of active lever presses during the last 2 days of SA at each dosage in both genders using twoway ANOVA. As shown in Figure 3, a significant effect of dosage $\left(\mathrm{F}_{3,44}=5.68, p<0.01\right)$ was found, whereas there was no significant difference between genders $\left(F_{1,44}=0.74\right.$, $p>0.05)$ nor was there a significant interaction between gender and dosage $\left(F_{2,44}=2.06, p>0.05\right)$. Post hoc testing (Fisher protected least significance) found that the $7.5 \mu \mathrm{g} / \mathrm{kg}$ group was significantly different from the 15 and $30 \mu \mathrm{g} / \mathrm{kg}$ groups ( $p<0.01$ for both comparisons). No other comparisons were significant $(p>0.05)$.

\section{Cumulative Daily Nicotine SA in Both Genders}

Representative cumulative daily active lever press records are shown for two female and two male adolescents that received $30 \mu \mathrm{g} / \mathrm{kg} /$ injection nicotine at FR 1 (Figure 4). In both adolescent females and males, active lever presses were 
$(92.5 \pm 2.7 \%$ and $92.3 \pm 8.0 \%$ for females and males,

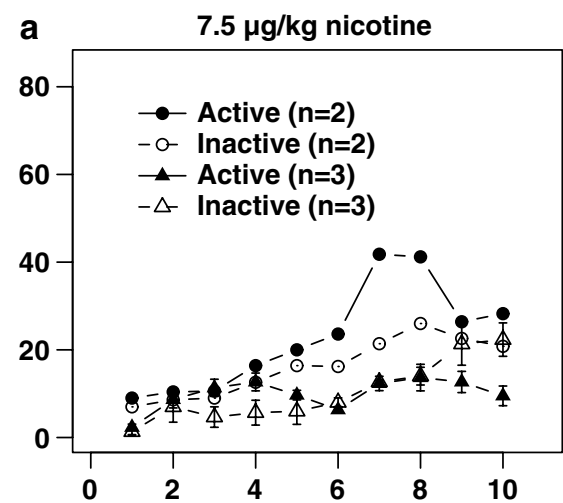
respectively).

\section{Nicotine SA with an Escalating FR Schedule in Female Adolescent Rats}

An escalating FR protocol was used to determine the reinforcing efficacy of nicotine under increasing workload (Figure 5). Data obtained on days 6-7 at each FR were used for statistical analysis, with the exception of FR 2, which was designed as a brief transition to the contingency of increasing FR; as the number of days in FR 2 were not sufficient to allow stable SA behavior, data obtained during this transition period were not analyzed. On average, rats self-administered $69 \pm 5,61 \pm 3,42 \pm 3$, and $20 \pm 2$ injections during the last 2 days under FR 1, FR 3, FR 5, and FR 7, respectively. The corresponding inactive lever presses were $24 \pm 2, \quad 34 \pm 3, \quad 24 \pm 2$ and $27 \pm 1$, respectively. ANOVA demonstrated an effect of FR on the number of injections obtained $\left(\mathrm{F}_{3,24}=5.48 ; p<0.01\right)$. Post hoc testing (Fisher's test of least significant difference (FLSD)) showed that the number of injections obtained at FR 1 was greater than FR 5 $(p<0.05)$ and FR $7 \quad(p<0.001)$. Thus, the reinforcing efficacy of nicotine, evidenced by the effect of increasing workload on the amount of nicotine obtained, was insufficient to maintain the SA behavior observed at FR 1 and 3 when FR was increased to 5 and 7.

\section{Nicotine SA in Female Adult vs Adolescent Rats}

Ten adult female Lewis rats acquired nicotine SA at $30 \mu \mathrm{g} /$ $\mathrm{kg} /$ injection, and these data were compared to those from adolescent females. Similar to our previous reports on adult male Lewis rats, adult females gradually acquired nicotine SA during the first week, thereafter stabilizing at greater than 40 injections/day (Figure 6). Repeated measures ANOVA found a significant main effect of lever $\left(\mathrm{F}_{1,18}=7.05, p<0.05\right)$, demonstrating the specificity of the active lever press behavior. The effect of day was also significant $\left(\mathrm{F}_{1,18}=6.19, p<0.001\right)$, and there was a significant day $\times$ lever interaction $\left(\mathrm{F}_{9,81}=3.57, p<0.001\right)$. When compared to adolescent females with access to the same dose of nicotine, repeated measures ANOVA found a significant main effect of age $\left(\mathrm{F}_{1,16}=8.57, p<0.01\right)$. The rate of acquisition, calculated as the slope of the linear regression of daily injection by day for the first 7 days, was compared according to Zar (1984). A statistically significant difference was found between the adolescents and adults $(p<0.001)$. Therefore, by day 10 , adolescent females not only self-administered significantly more nicotine than adult females, but they also acquired the behavior at an accelerated rate.

\section{DISCUSSION}

The definition of adolescence in rodents is controversial, and generally varies according to the objectives of a particular study. Based on a conservative perspective in rodents (Spear, 2000), prototypical adolescent changes occur from approximately PN 28-42, although some developmental changes specific to adolescence do persist through PN 55 (Spear, 2000). The rats used in these $15 \mathrm{~min}$. The majority of the active lever presses occurred during the dark phase of the light cycle in both genders 


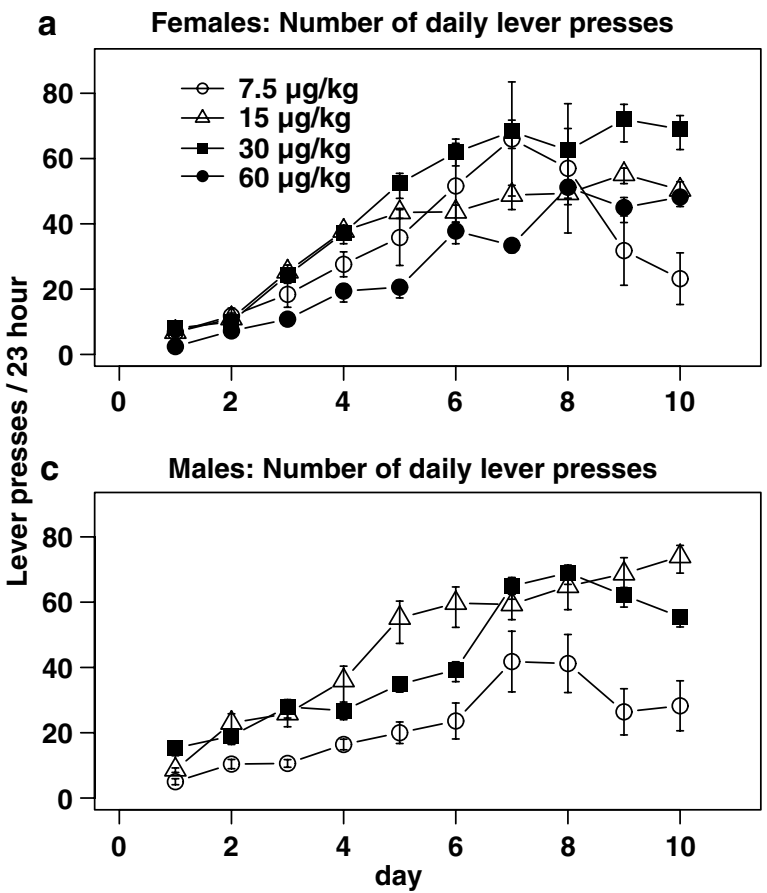

b Females: Average number of lever presses (d9-10)

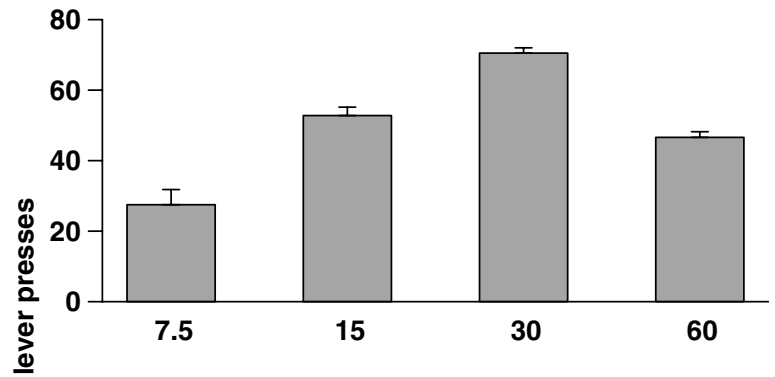

d Males: Average number of lever presses (d9-10)

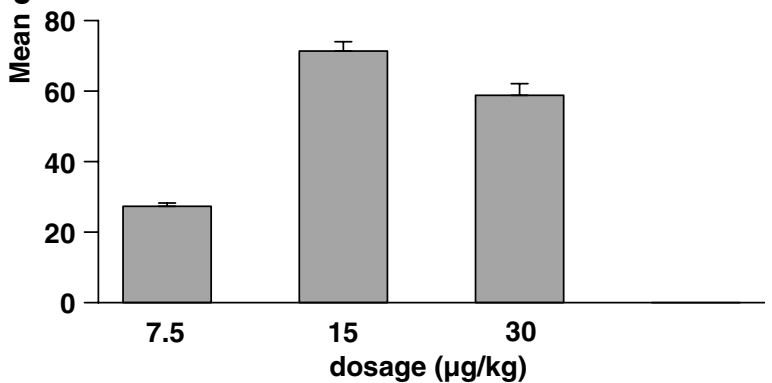

Figure 3 Effect of nicotine dosage on SA in adolescent rats. The effect of nicotine dosage on daily active lever presses in each gender is shown in (a) and (c). Two-way ANOVA was performed to analyze the effect of nicotine dosage and gender on the number of active lever presses (average of days 9 and I0). This showed a significant effect of dosage $\left(F_{3,44}=5.68, p<0.0 \mathrm{I}\right)(\mathrm{b}, \mathrm{d})$. Post hoc Fisher protected least significance test found that the $7.5 \mu \mathrm{g} / \mathrm{kg}$ group was significantly less than the 15 and $30 \mu \mathrm{g} / \mathrm{kg}$ groups in both genders $(p<0.0 \mathrm{I}$ for both dosages).

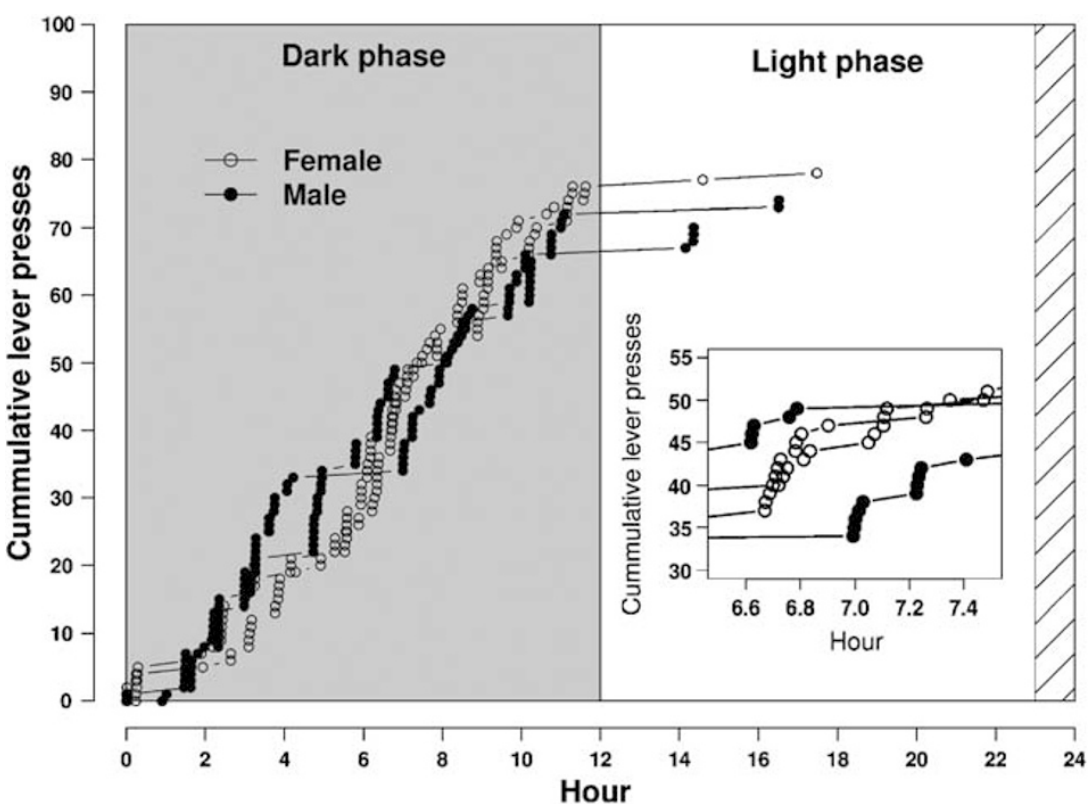

Figure 4 Cumulative nicotine SA in both genders on day 10. Representative cumulative daily active lever press records are shown for two female and two male adolescents receiving $30 \mu \mathrm{g} / \mathrm{kg} /$ injection nicotine at FR I on SA day I0. In both genders, active lever presses were clustered, generally in groups of 3-5 lever presses every 10-15 min (see inset). The majority of the active lever presses occurred during the dark phase of the light cycle $(92.5 \pm 2.7$ and $92.3 \pm 8.0 \%$ for females and males, respectively).

experiments first had access to nicotine by PN 43-45, and were PN 53-55 at the end of the 10-day dose-response studies. Thus, the present nicotine SA experiments were performed within the age range of adolescence, as broadly defined. The present study demonstrates that mid-adolescent Lewis rats of both genders rapidly learned to self- administer i.v. nicotine at dosages between 15 and $60 \mu \mathrm{g} / \mathrm{kg} /$ injection when prolonged access $(23 \mathrm{~h} /$ day) was allowed. This was achieved without prior shaping, conditioning, priming, or food deprivation. The preponderance of nicotine SA behavior was restricted to the dark phase of the light cycle, when rodents are most active. 


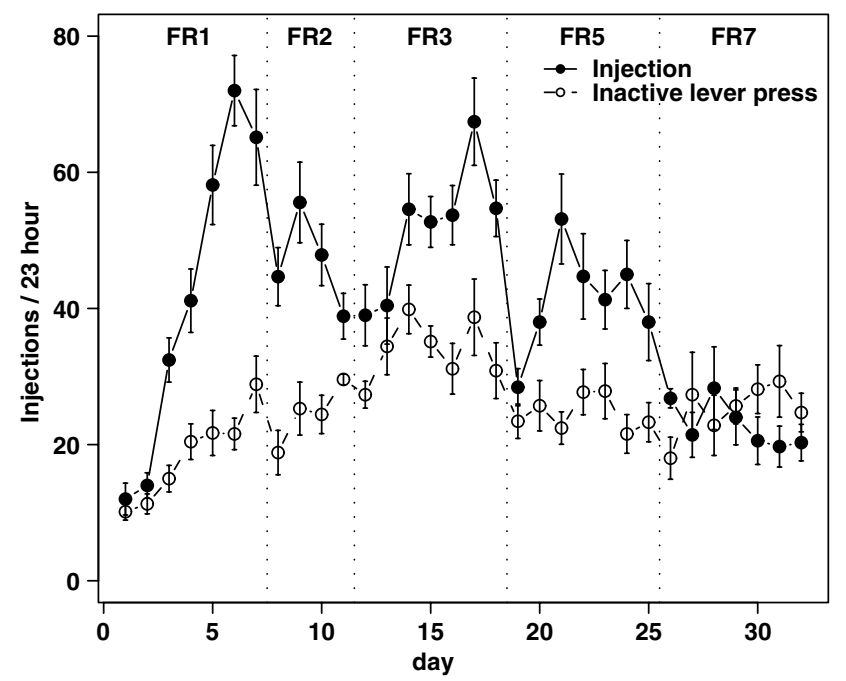

Figure 5 Nicotine SA under an escalating FR schedule in female adolescent rats. An escalating FR protocol was used to determine the effect of increasing workload on nicotine SA. Data obtained during SA days 6-7 at each FR were used for statistical analysis (except FR 2, designed to be a brief transition to the contingency of increased FR). Rats self-administered $69 \pm 5,61 \pm 3,42 \pm 3$, and $20 \pm 2$ injections of nicotine ( $30 \mu \mathrm{g} / \mathrm{kg} /$ injection) during the last 2 days under FR I, FR 3, FR 5, and FR 7, respectively. ANOVA demonstrated an effect of FR on the number of injections obtained $\left(F_{3,24}=5.48 ; p<0.0 \mathrm{I}\right)$. Post hoc testing $(\mathrm{LSD})$ showed that the number of injections obtained at FR I was greater than FR $5(p<0.05)$ and FR $7(p<0.001)$.

Acquisition of nicotine SA was tested with four dosages ranging from 7.5 to $60 \mu \mathrm{g} / \mathrm{kg} /$ injection in female adolescent rats. The number of injections self-administered under different dosages showed an inverse U-shaped relationship. Similar results were obtained using male adolescent rats. Thus, it appears that animals were attempting to regulate their drug intake by reducing their SA rates at higher dosages. However, their ability to titrate their intake was limited in that a greater total daily amount of nicotine was self-administered at higher dosages. These data are very similar to previous findings in adult male Lewis rats (Valentine et al, 1997), adult Sprague-Dawley rats (Donny et al, 2000), as well as in mice that voluntarily consume nicotine in drinking water (Klein et al, 2004). In addition, these data suggest that $15-30 \mu \mathrm{g} / \mathrm{kg} /$ injection nicotine is within the optimal dose range for SA in adolescent Lewis rats.

There are at least two previous studies on nicotine SA by adolescent rats; both of these used limited access models, permitting SA for $1-3 \mathrm{~h}$ per day. Levin et al (2003) initially trained PN 34 female rats to lever press for food. Those rats meeting criteria then received both food and nicotine for 34 days before nicotine alone, beginning at PN 43-49. Rats self-administered approximately 13 injections per session when nicotine was the sole reinforcer. However, data on lever press activity during sequential individual SA sessions were not reported, and, therefore, the stability of the nicotine SA behavior cannot be evaluated. In addition, the frequency of inactive lever presses was not reported; as such, the specificity of the nicotine-reinforced active lever press behavior was not established definitively.

In a second study (Belluzzi et al, 2005), PN 27-31 male rats, without prior training, received i.v. nicotine $(30 \mu \mathrm{g} / \mathrm{kg})$

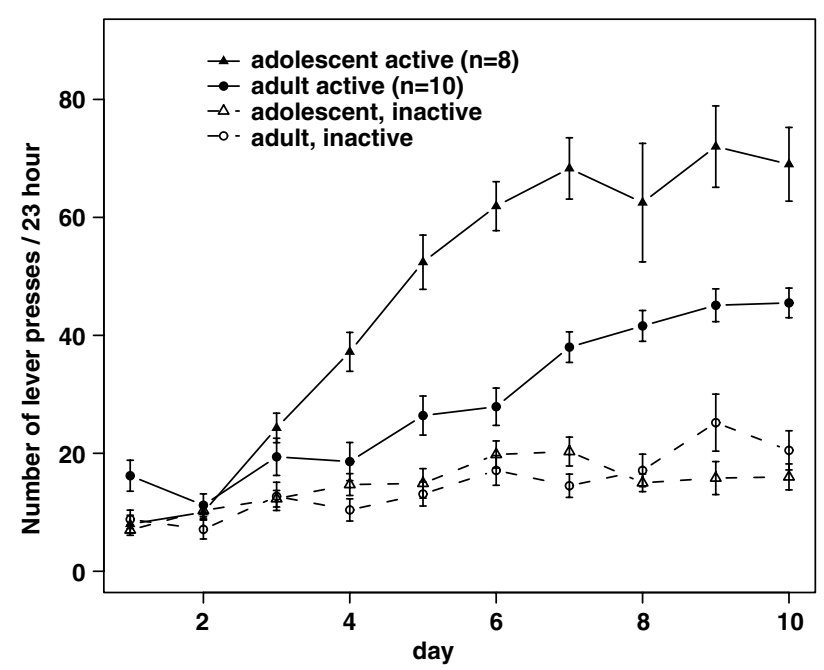

Figure 6 Nicotine SA in female adolescent vs adult rats. As observed in female adolescent Lewis rats, female adults $(n=10)$ acquired stable SA when given access to $30 \mu \mathrm{g} / \mathrm{kg}$ nicotine. The rate of acquisition, calculated as the slope of the linear regression of daily injection by day for the first 7 days, was statistically different in adolescents vs adults $(p<0.001)$. In addition, the number of active lever presses by female adult rats during the last 2 days of SA, when the behavior was stable, was significantly less than that in female adolescents $(p<0.01)$. Therefore, female adolescents not only acquired nicotine SA at faster rates, but they also self-administered nicotine at significantly higher levels than female adults.

when an appropriate nose-poke was performed. During the 5 day test interval of acquisition (one $3 \mathrm{~h}$ session/day), no significant day-by-day change in nose-poke behavior occurred. Adult rats also failed to self-administer nicotine under these conditions. However, nicotine SA was only observed in these adolescents when acetaldehyde was coadministered with nicotine. The difference in the efficacy of nicotine to elicit nose poke $v s$ lever press behaviors may reflect intrinsic differences in the ability to learn the association between nicotine reinforcement and an ethologically encoded behavior compared to a novel behavior. Additionally, the capacity of very young adolescents (ie PN 27-31) to learn nicotine SA in a lever press model is presently unknown.

In contrast to these two limited-access studies, the experiments presented herein demonstrate adolescent nicotine SA in PN 43-45 rats, based on the following: (a) active lever presses increased gradually during the first 3-4 days of acquisition (Figures 1 and 2); (b) active $v s$ inactive lever press behavior was maintained at ratios greater than 2 across all dosages within 3-4 days of initial access to nicotine, indicating the specificity of the nicotine-reinforced behavior; (c) at each effective dosage $(15-60 \mu \mathrm{g} / \mathrm{kg} /$ injection), a similar number of nicotine injections per day were self-administered during the last 3-4 days at that dosage, demonstrating the stability of the nicotine SA behavior (Figures 1 and 2). Thus, data presented herein are likely to be the first report of nicotine SA in adolescent rats in which the specificity and stability of the behavior were clearly demonstrated in both genders.

Several model-dependent factors may have contributed to differences between the results of the present study and previous reports. In this study, rats were given access to nicotine for $23 \mathrm{~h} /$ day, whereas the drug was available for 
only $1-3 \mathrm{~h}$ in the limited-access models. Our laboratory and others have shown, using this prolonged access model (Valentine et al, 1997; Fu et al, 2001, 2003; Brower et al, 2002; LeSage et al, 2002, 2003, 2006; Parker et al, 2004), that adult rats will acquire nicotine $\mathrm{SA}$ in the absence of food deprivation or prior food-reinforced conditioning. For several reasons, this prolonged access model is advantageous for studying nicotine $\mathrm{SA}$ in rats during midadolescence. First, rodent adolescence is relatively brief (eg from PN 22 to 55); therefore, the time available for shaping and/or conditioning is limited. Thus, the time required for rats to first acquire food-reinforced lever press behavior would significantly reduce the time available to learn nicotine SA during the window of adolescence. Second, in our pilot studies (data not shown), the rapid growth in body size during early adolescence displaced the nicotine-delivering jugular catheter in many animals, precluding the reliable study of nicotine SA during early adolescence. Third, food restriction in adolescent rats restricts growth and delays puberty (Delemarre-van de Waal et al, 2002), which may affect the performance of nicotine SA behavior. Food restriction also elevates plasma corticosterone (Marinelli et al, 1996), which enhances the activity of the underlying dopaminergic reward system (Barrot et al, 2000; Marinelli and Piazza, 2002). Therefore, animals trained using food restriction demonstrated enhanced motivation (Carroll and Lac, 1993; Piazza and Le Moal, 1996) when nicotine first became available. This confounding factor precludes the possibility of attributing the acquisition of SA behavior to nicotine per se.

Two additional factors distinguish the present study from these previous reports on adolescent nicotine SA. First, the Lewis strain of rat differentiates our studies from those conducted with Sprague-Dawley rats. We have previously reported that adult Lewis rats are more prone to nicotine SA compared to other rat strains, including Sprague-Dawley (Brower et al, 2002). Similar results have been reported for other drugs of abuse (Kosten and Ambrosio, 2002). Although those data were obtained using adult rats, genetically determined predisposition is likely to be maintained from adolescence to adulthood within the same strain. Another difference that may potentially impact nicotine SA is the absence of cues (eg tones) paired with the delivery of nicotine in this study; such cues were used in both of the aforementioned reports. Although cues have been shown to facilitate the acquisition of nicotine SA in adult rats (Caggiula et al, 2002a, b), their potential effect on the initial acquisition of nicotine $\mathrm{SA}$ in adolescent rats is unknown.

We found that adolescent rats acquired nicotine SA at an accelerated rate and also attained higher levels of stable nicotine SA than adult rats. The increased rate of nicotine metabolism in adolescents, reported by Trauth et al (2000b), might contribute to these behavioral observations. In adolescent rats chronically infused with nicotine, plasma nicotine levels were significantly lower than in adults (Trauth et al, 2000b). It is therefore possible that adolescent and adult rats self-administer different amounts in order to achieve similar nicotine plasma levels. Both behavioral theory and neurochemical observations indicate that increased active lever presses per se, apart from plasma nicotine levels, are significant determinants of the strength of associative learning. Even if plasma levels were the same in female adolescents and adults, the behavioral data demonstrate that a significantly greater number of active lever responses would be required to achieve such nicotine plasma levels in adolescents compared to adults. This would yield more stimulus-response (SR) associations ( $\mathrm{S}=$ cue light/lever and $\mathrm{R}=$ active lever presses), producing stronger operant conditioning in adolescents. In vivo voltammetry studies provide insight into the neurochemical correlates of this behavior. As an animal approaches the active lever and/ or presses that lever, dopamine is released (Phillips et al, 2003) and specific neurons activated (Carelli and Deadwyler, 1994) in the nucleus accumbens (NAcc) before receiving a drug reinforcer. Thus, the conditioned stimulus properties of the lever itself are sufficient to elicit dopamine release. This implies that the conditioned behavioral response of increased bar pressing per se (ie in adolescents $v s$ adults) would be associated with increased ventral tegmental area neuronal activity and NAcc dopamine release. In addition to this increase in the level of active lever presses achieved by adolescents, the enhanced rate of learning during the acquisition phase of nicotine SA (ie day 1-day 5) suggests a propensity to develop greater nicotine dependence in the adolescent compared to adult. This enhanced rate of learning is evident before stable nicotine SA and nicotine plasma levels have been achieved.

Differences in stress responses may differentially affect the pattern of nicotine SA in adolescents compared to adults. Cruz et al (2005) reported that adult rats developed tolerance to nicotine-induced corticosterone release, whereas adolescents did not. Therefore, it is conceivable that the persistence of nicotine-induced corticosterone release may have enhanced the motivation (Marinelli et al, 1996) to self-administer nicotine in adolescent rats. In comparison to adults, adolescents also may have different behavioral responses to being housed individually. Although no direct evidence on nicotine SA is available, limited data on voluntary ethanol consumption (Doremus et al, 2005) suggest that adult rats were susceptible to isolation-induced suppression of ethanol intake, whereas adolescents were not. However, housing conditions only accounted for part of this difference in ethanol consumption. In addition, there are a manifold of central nervous system developmental changes that might have contributed to the adolescent pattern of nicotine SA (Spear, 2000). For example, a significant reduction in cortical synapses takes place during adolescence, yielding a net increase of inhibitory inputs to the adult cortex (Spear, 2000). Regardless of specific mechanism, the accelerated rate of acquisition by female adolescents compared to adults suggests that stronger learning may occur in adolescents, based on more stimulus-reward associations formed on a day-to-day basis. In humans, such enhanced learning could be related to the strong physical dependence that develops in adolescent smokers and their subsequent failure to successfully quit smoking as adults.

In conclusion, the present study demonstrates that midadolescent Lewis rats rapidly learn to self-administer i.v. nicotine at dosages from 15 to $60 \mu \mathrm{g} / \mathrm{kg} /$ injection in both genders, when prolonged access $(23 \mathrm{~h} /$ day) is provided. Mid-adolescent Lewis rats learn this without prior shaping, conditioning, priming, or coinjections of other agents (eg 
acetaldehyde). The effective nicotine dosage range found in these studies is similar to those reported in both prolonged and limited-access models of nicotine SA in adult rats. As previously reported in adult rats, the reinforcing efficacy of nicotine, as evidenced by the effect of increasing workload on the amount of nicotine obtained, declined at FR 5 and 7 in adolescents. Finally, adolescent rats acquired nicotine $\mathrm{SA}$ at a faster rate and attained higher levels of stable SA than adults.

\section{ACKNOWLEDGEMENTS}

This research was supported by DA-03977 (BMS) and DA015525 (SGM). We thank Victoria Kane, $\mathrm{PhD}$ for her pilot study, and thank Weihua Cheng, Wenbo Ge, and Fan Wang for their assistance.

\section{REFERENCES}

Barrot M, Marinelli M, Abrous DN, Rouge-Pont F, Le Moal M, Piazza PV (2000). The dopaminergic hyper-responsiveness of the shell of the nucleus accumbens is hormone-dependent. Eur J Neurosci 12: 973-979.

Belluzzi JD, Lee AG, Oliff HS, Leslie FM (2004). Age-dependent effects of nicotine on locomotor activity and conditioned place preference in rats. Psychopharmacology (Berlin) 174: 389-395.

Belluzzi JD, Wang R, Leslie FM (2005). Acetaldehyde enhances acquisition of nicotine self-administration in adolescent rats. Neuropsychopharmacology 30: 705-712.

Brower VG, Fu Y, Matta SG, Sharp BM (2002). Rat strain differences in nicotine self-administration using an unlimited access paradigm. Brain Res 930: 12-20.

Caggiula AR, Donny EC, Chaudhri N, Perkins KA, Evans-Martin FF, Sved AF (2002a). Importance of nonpharmacological factors in nicotine self-administration. Physiol Behav 77: 683-687.

Caggiula AR, Donny EC, White AR, Chaudhri N, Booth S, Gharib MA et al (2002b). Environmental stimuli promote the acquisition of nicotine self-administration in rats. Psychopharmacology (Berlin) 163: 230-237.

Carelli RM, Deadwyler SA (1994). A comparison of nucleus accumbens neuronal firing patterns during cocaine self-administration and water reinforcement in rats. J Neurosci 14: 77357746.

Carroll ME, Lac ST (1993). Autoshaping i.v. cocaine selfadministration in rats: effects of nondrug alternative reinforcers on acquisition. Psychopharmacology (Berlin) 110: 5-12.

Chen J, Millar WJ (1998). Age of smoking initiation: implications for quitting. Health Rep 9: 39-46 (Eng); 39-48(Fre).

Corrigall WA, Coen KM (1989). Nicotine maintains robust selfadministration in rats on a limited-access schedule. Psychopharmacology (Berlin) 99: 473-478.

Cox BM, Goldstein A, Nelson WT (1984). Nicotine self-administration in rats. Br J Pharmacol 83: 49-55.

Cruz FC, Delucia R, Planeta CS (2005). Differential behavioral and neuroendocrine effects of repeated nicotine in adolescent and adult rats. Pharmacol Biochem Behav 80: 411-417.

Delemarre-van de Waal HA, van Coeverden SC, Engelbregt MT (2002). Factors affecting onset of puberty. Horm Res 57(Suppl 2): 15-18.

Donny EC, Caggiula AR, Knopf S, Brown C (1995). Nicotine self-administration in rats. Psychopharmacology (Berlin) 122: 390-394.

Donny EC, Caggiula AR, Rowell PP, Gharib MA, Maldovan V, Booth $S$ et al (2000). Nicotine self-administration in rats: estrous cycle effects, sex differences and nicotinic receptor binding. Psychopharmacology (Berlin) 151: 392-405.
Doremus TL, Brunell SC, Rajendran P, Spear LP (2005). Factors influencing elevated ethanol consumption in adolescent relative to adult rats. Alcohol Clin Exp Res 29: 1796-1808.

Eissenberg T, Balster RL (2000). Initial tobacco use episodes in children and adolescents: current knowledge, future directions. Drug Alcohol Depend 59(Suppl 1): S41-S60.

Everett SA, Warren CW, Sharp D, Kann L, Husten CG, Crossett LS (1999). Initiation of cigarette smoking and subsequent smoking behavior among U.S. high school students. Prev Med 29: 327333.

Faraday MM, Elliott BM, Grunberg NE (2001). Adult vs adolescent rats differ in biobehavioral responses to chronic nicotine administration. Pharmacol Biochem Behav 70: 475-489.

Faraday MM, Elliott BM, Phillips JM, Grunberg NE (2003). Adolescent and adult male rats differ in sensitivity to nicotine's activity effects. Pharmacol Biochem Behav 74: 917-931.

Fernandez E, Schiaffino A, La Vecchia C, Borras JM, Nebot M, Salto E et al (1999). Age at starting smoking and number of cigarettes smoked in Catalonia, Spain. Prev Med 28: 361-366.

Fu Y, Matta SG, Brower VG, Sharp BM (2001). Norepinephrine secretion in the hypothalamic paraventricular nucleus of rats during unlimited access to self-administered nicotine: an in vivo microdialysis study. J Neurosci 21: 8979-8989.

Fu Y, Matta SG, Kane VB, Sharp BM (2003). Norepinephrine release in amygdala of rats during chronic nicotine selfadministration: an in vivo microdialysis study. Neuropharmacology 45: 514-523.

Glynn TJ, Greenwald P, Mills SM, Manley MW (1993). Youth tobacco use in the United States - problem, progress, goals, and potential solutions. Prev Med 22: 568-575.

Goldberg SR, Spealman RD, Goldberg DM (1981). Persistent behavior at high rates maintained by intravenous self-administration of nicotine. Science 214: 573-575.

Hanson HM, Ivester CA, Morton BR (1979). Nicotine selfadministration in rats. NIDA Res Monogr 23: 70-90.

Jaffe JH, Kanzler MB (1978). Tobacco and nicotine self-administration in humans: the evolution of a methodology. NIDA Res Monogr 20: 209-220.

Kelley AE, Schochet T, Landry CF (2004). Risk taking and novelty seeking in adolescence: introduction to part I. Ann NY Acad Sci 1021: 27-32.

Klein LC, Stine MM, Vandenbergh DJ, Whetzel CA, Kamens HM (2004). Sex differences in voluntary oral nicotine consumption by adolescent mice: a dose-response experiment. Pharmacol Biochem Behav 78: 13-25.

Kosten TA, Ambrosio E (2002). HPA axis function and drug addictive behaviors: insights from studies with Lewis and Fischer 344 inbred rats. Psychoneuroendocrinology 27: 35-69.

LeSage MG, Keyler DE, Collins G, Pentel PR (2003). Effects of continuous nicotine infusion on nicotine self-administration in rats: relationship between continuously infused and selfadministered nicotine doses and serum concentrations. Psychopharmacology (Berlin) 170: 278-286.

Lesage MG, Keyler DE, Hieda Y, Collins G, Burroughs D, Le C et al (2006). Effects of a nicotine conjugate vaccine on the acquisition and maintenance of nicotine self-administration in rats. Psychopharmacology (Berlin) 184: 409-416.

LeSage MG, Keyler DE, Shoeman D, Raphael D, Collins G, Pentel PR (2002). Continuous nicotine infusion reduces nicotine selfadministration in rats with $23-\mathrm{h} /$ day access to nicotine. Pharmacol Biochem Behav 72: 279-289.

Levin ED, Rezvani AH, Montoya D, Rose JE, Swartzwelder HS (2003). Adolescent-onset nicotine self-administration modeled in female rats. Psychopharmacology (Berlin) 169: 141-149.

Marinelli M, Le Moal M, Piazza PV (1996). Acute pharmacological blockade of corticosterone secretion reverses food restrictioninduced sensitization of the locomotor response to cocaine. Brain Res 724: 251-255. 
Marinelli M, Piazza PV (2002). Interaction between glucocorticoid hormones, stress and psychostimulant drugs. Eur J Neurosci 16: 387-394.

O’Dell LE, Bruijnzeel AW, Ghozland S, Markou A, Koob GF (2004). Nicotine withdrawal in adolescent and adult rats. Ann NY Acad Sci 1021: 167-174.

Parker SL, Fu Y, McAllen K, Luo J, McIntosh JM, Lindstrom JM et al (2004). Up-regulation of brain nicotinic acetylcholine receptors in the rat during long-term self-administration of nicotine: disproportionate increase of the alpha6 subunit. $\mathrm{Mol}$ Pharmacol 65: 611-622.

Phillips PE, Stuber GD, Heien ML, Wightman RM, Carelli RM (2003). Subsecond dopamine release promotes cocaine seeking. Nature 422: 614-618.

Piazza PV, Le Moal ML (1996). Pathophysiological basis of vulnerability to drug abuse: role of an interaction between stress, glucocorticoids, and dopaminergic neurons. Annu Rev Pharmacol Toxicol 36: 359-378.

Rasmussen T, Swedberg MD (1998). Reinforcing effects of nicotinic compounds: intravenous self-administration in drugnaive mice. Pharmacol Biochem Behav 60: 567-573.

Rigotti NA (1990). How can we help the remaining smokers to quit? Am J Prev Med 6: 249-250.

Risner ME, Goldberg SR (1983). A comparison of nicotine and cocaine self-administration in the dog: fixed-ratio and progressive-ratio schedules of intravenous drug infusion. J Pharmacol Exp Ther 224: 319-326.

Rose JE, Behm FM, Westman EC, Bates JE (2003). Mecamylamine acutely increases human intravenous nicotine self-administration. Pharmacol Biochem Behav 76: 307-313.

SAMHSA (2002). National Survey on Drug Use and Health. US Public Health Service: Rockville, MD.
Schochet TL, Kelley AE, Landry CF (2004). Differential behavioral effects of nicotine exposure in adolescent and adult rats. Psychopharmacology (Berlin) 175: 265-273.

Schoffelmeer AN, De Vries TJ, Wardeh G, van de Ven HW, Vanderschuren LJ (2002). Psychostimulant-induced behavioral sensitization depends on nicotinic receptor activation. J Neurosci 22: 3269-3276.

Slawecki CJ, Ehlers CL (2002). Lasting effects of adolescent nicotine exposure on the encephalogram. Event related potentials, and locomotor activity in the rat. Brain Res Dev Brain Res 138: $15-25$.

Slotkin TA (2002). Nicotine and the adolescent brain: insights from an animal model. Neurotoxicol Teratol 24: 369-384.

Spear LP (2000). The adolescent brain and age-related behavioral manifestations. Neurosci Biobehav Rev 24: 417-463.

Stolerman IP, Jarvis MJ (1995). The scientific case that nicotine is addictive. Psychopharmacology (Berlin) 117: 2-10; discussion 14-20.

Trauth JA, Seidler FJ, Slotkin TA (2000a). Persistent and delayed behavioral changes after nicotine treatment in adolescent rats. Brain Res 880: 167-172.

Trauth JA, Seidler FJ, Slotkin TA (2000b). An animal model of adolescent nicotine exposure: effects on gene expression and macromolecular constituents in rat brain regions. Brain Res 867: 29-39.

Valentine JD, Hokanson JS, Matta SG, Sharp BM (1997). Selfadministration in rats allowed unlimited access to nicotine. Psychopharmacology (Berlin) 133: 300-304.

Vastola BJ, Douglas LA, Varlinskaya EI, Spear LP (2002). Nicotineinduced conditioned place preference in adolescent and adult rats. Physiol Behav 77: 107-114.

Zar JH (1984). Biostatistical Analysis. Prentice-Hall: New Jersey. 